\title{
Tendências temporais de índices de vegetação nos campos do Pampa do Brasil e do Uruguai
}

\author{
Ana Paula Luz Wagner( ${ }^{(1)}$, Denise Cybis Fontana( ${ }^{(2)}$, Clyde Fraisse ${ }^{(3)}$, Eliseu José Weber ${ }^{(4)}$ \\ e Heinrich Hasenack ${ }^{(4)}$
}

\begin{abstract}
(1)Universidade Federal do Rio Grande do Sul (UFRGS), Centro Estadual de Pesquisas em Sensoriamento Remoto e Meteorologia, Avenida Bento Gonçalves, no 9.500, Caixa Postal 15044, CEP 91501-970 Porto Alegre, RS. E-mail: anaplw@gmail.com (2)UFRGS, Departamento de Plantas Forrageiras e Agrometeorologia, Avenida Bento Gonçalves, no 7.712, Caixa Postal 15100, CEP 91501-970 Porto Alegre, RS. E-mail: dfontana@ufrgs.br (3)Florida University, Department of Agricultural and Biological Engineering, 239, Rogers Hall, P.O. Box 110570, Gainesville, FL 32611-0570, EUA. E-mail: cfraisse@ufl.edu (4)UFRGS, Centro de Ecologia, Caixa Postal 15007, CEP 91501-970 Porto Alegre, RS. E-mail: eliseu.weber@ufrgs.br, hhasenack@ufrgs.br
\end{abstract}

Resumo - O objetivo deste trabalho foi avaliar a redução do vigor vegetativo da cobertura vegetal do Pampa do Brasil e do Uruguai, por meio da identificação de tendências negativas em séries temporais de imagens. Utilizaram-se séries temporais de imagens de NDVI/EVI do sensor Modis, de 2000 a 2011; imagens de índices de umidade do solo do "climate forecast system reanalysis"; e dados de precipitação pluvial de estações meteorológicas. O estudo quantificou tendências lineares e não lineares nas séries de NDVI e EVI, em áreas de campos. Na tendência monotônica de Mann-Kendall, a 5\% de probabilidade, $81,9 \%$ da área total estudada foi significativa com o NDVI, e 74,8\%, com o EVI; no entanto, o EVI apresentou contraste superior na estimativa dos parâmetros. Os resultados mostraram maior sinal negativo a oeste, com valores médios de $\mathrm{R}^{2}>0,15, \mathrm{r}<-0,3 \mathrm{e}$ $\tau<-0,15$ na tendência dos índices de vegetação, e tendência decrescente para NDVI, EVI e precipitação pluvial, com menores valores médios de umidade do solo. A tendência negativa dos índices de vegetação, relacionada à combinação da ocorrência de deficit hídrico em solos rasos com o sobrepastoreio, indica alterações no padrão de cobertura vegetal do Pampa, com redução do vigor vegetativo.

Termos para indexação: campos nativos, EVI, Modis, NDVI, séries temporais.

\section{Temporal trends of vegetation indices on Pampa grasslands in Brazil and Uruguay}

\begin{abstract}
The objective of this work was to evaluate the reduction in the vegetative vigor of Pampa vegetation cover in Brazil and Uruguay, by identifying negative trends in images time series. The following were used: time series of NDVI/EVI images from the Modis sensor, from 2000 to 2011; images from soil moisture indices from the climate forecast system reanalysis; and precipitation data from meteorological stations. The study quantified linear and nonlinear trends in the NDVI and EVI series in grassland areas. With the MannKendall monotonic trend, at $5 \%$ probability, $81.9 \%$ of the total area studied was significant with NDVI, and $74.8 \%$ with EVI; however, EVI showed superior contrast in the estimation of parameters. The results showed: highest negative signal in the west, with average values of $\mathrm{R}^{2}>0.15, \mathrm{r}<-0.3$, and $\tau<-0.15$ in the tendency of the vegetation indices; and decreasing tendency for NDVI, EVI, and rainfall, with lower mean soil moisture values. The negative trend of the vegetation indices, related to the combination of drought occurrence in surface soils with excessive grazing, indicates changes in the pattern of Pampa vegetation cover, with reduction in vegetative vigor.
\end{abstract}

Index terms: natural grasslands, EVI, Modis, NDVI, time series.

\section{Introdução}

Os campos que cobrem a porção de terra no extremo Sul do Brasil e no Uruguai pertencem a um complexo de áreas abertas que se estendem até a Argentina e o Paraguai (Crawshaw et al., 2007), conhecido como Pampa. Na República Oriental do
Uruguai, coberta por vegetação predominantemente campestre, os processos de intervenção na última década ocasionaram a diminuição de quase 110 mil hectares por ano de campos naturais (Jaurena et al., 2013), enquanto, no Estado do Rio Grande do Sul, restam apenas $50 \%$ de sua área original (Pillar \& Vélez, 2010). 
Os campos sofreram acentuada descaracterização, principalmente pela forte expansão agrícola ocorrida na década de 1970 e, recentemente, pela conversão de áreas campestres em monoculturas de Pinus sp., Acacia spp. e Eucalytus spp. (Berreta, 2009), bem como pelo excesso de lotação empregado na exploração pecuária (Carvalho et al., 2006).

Além destes fatores, as áreas campestres, principal componente da região, podem sofrer modificação nos padrões de resposta da cobertura vegetal ao longo do tempo, em decorrência de eventos climáticos severos, ciclos fenológicos anuais e eventos episódicos, como é o caso do El Niño Oscilação Sul (Enos). O Enos tem forte influência na região, em razão do efeito que este fenômeno provoca sobre a precipitação pluvial regional (Jacóbsen et al., 2004). O status, ou a condição da vegetação, fornece indicativos ambientais importantes, de modo que declínios e tendências temporais são considerados indicadores de degradação da terra (Jong \& Bruin, 2012).

De forma geral, os índices de vegetação, definidos como adimensionais, são medidas radiométricas que funcionam como indicadores da abundância relativa e da atividade da vegetação verde (Ji et al., 2011), e suas tendências podem ser usadas como indicativos ecológicos (Jong \& Bruin, 2012). A estreita correlação entre dinâmica espaço-temporal da vegetação e índices de vegetação mostra o seu potencial para o monitoramento da cobertura vegetal sobre áreas extensas (Risso et al., 2012). Entre esses índices, destacam-se o "normalized difference vegetation index" (NDVI), baseado na refletância do vermelho e do infravermelho (Rouse et al., 1974); e o "enhanced vegetation index" (EVI), que inclui a refletância do azul (Huete et al., 1997).

Estudos têm analisado as tendências dos índices de vegetação, especialmente do NDVI, para inúmeros fins, desde mudanças de uso e cobertura do solo a mudanças fenológicas (Leeuwen et al., 2006). Uma abordagem frequente é estabelecer tendências por meio de regressão linear do NDVI, calculado em períodos anuais ou sazonais (Jong \& Bruin, 2012; Forkel et al., 2013). A regressão linear é um teste paramétrico que pode apresentar, entretanto, dificuldades na caracterização de tendências, referentes à capacidade de se determinar o quanto o coeficiente de declividade da reta ajustada difere significativamente de zero. Além disso, a capacidade de detectar tendências na razão de crescimento ou intensidade na vegetação em períodos sazonais, muitas vezes, é ocultada quando os dados de NDVI são integrados em frequências anuais (Jong \& Bruin, 2012).

Em séries temporais de imagens de índices de vegetação, a autocorrelação no conjunto de dados pode se tornar um problema, à medida que causa, nos processos de regressões lineares, diminuição nos valores esperados das tendências. No caso da não remoção da sazonalidade dos dados, é necessário aplicar testes de tendência não paramétricos, os quais levam em conta a sazonalidade da série (Neeti \& Eastman, 2011).

O objetivo deste trabalho foi avaliar a redução do vigor vegetativo da cobertura vegetal do Pampa do Brasil e do Uruguai, por meio da identificação de tendências negativas em séries temporais de imagens NDVI e EVI do sensor Modis.

\section{Material e Métodos}

A área experimental compreende a porção do Pampa que abrange a República Oriental do Uruguai e a porção meridional do Estado do Rio Grande do Sul (latitudes $27^{\circ}$ e $34^{\circ} 30^{\prime} \mathrm{S}$ e longitudes $48^{\circ} 30^{\prime}$ e $58^{\circ} 55^{\prime} \mathrm{W}$ ) (Figura 1).

Foram utilizadas séries temporais de imagens de NDVI e EVI, versão V005 (Huete et al., 2002), do período de $18 / 2 / 2000$ a $31 / 10 / 2011$, o que totalizou 269 datas de imagens. Os dados apresentam resolução temporal de 16 dias e resolução espacial de $250 \mathrm{~m}$, e foram obtidos do banco de dados do "Earth observing system data and information system" da Nasa (Eosdis/ Nasa). O processamento foi executado no programa Envi 4.2 + IDL (Exelis, McLean, VA, EUA).

Além das imagens dos índices NDVI e EVI, também foram utilizadas imagens de índices de umidade do solo, calculada para as profundidades de 40 e $100 \mathrm{~cm}$, para o mesmo período, o que totalizou 4.383 datas. O produto é o resultado da aplicação do modelo de superfície terrestre Noah LSM (Ek et al., 2003) implementado no acoplamento terra-atmosferaoceano e semiacoplado ao "climate forecast system reanalysis" (CFSR) e ao "global land data assimilation system" (GLDAS) (Saha et al., 2010). A série temporal tem cobertura global diária com resolução espacial de $0,25^{\circ}$. Os dados foram obtidos do "National Centers for Environmental Prediction" (NCEP) e do CFSR, e transformados do formato "Fortran 4-byte littleendian" para imagem.

Pesq. agropec. bras., Brasília, v.48, n.9, p.1192-1200, set. 2013 DOI: 10.1590/S0100-204X2013000900002 
Também foram utilizados dados meteorológicos diários de precipitação pluvial de três estações meteorológicas disponíveis na área de estudo, escolhidas em razão do menor índice de umidade do solo, próximas dos campos de solos rasos, região de maior sinal negativo nas tendências, a saber: Quaraí e Santana do Livramento, no Estado do Rio Grande do Sul; e Salto, no Uruguai. Os dados do Brasil foram disponibilizados pela Fundação Estadual de Pesquisa Agropecuária (Fepagro) e pelo Instituto Nacional de Meteorologia (INMET), e os dados do Uruguai, pelo Instituto Nacional de Investigación Agropecuaria (INIA).

As estimativas das tendências e dos níveis de significância das séries temporais dos índices de vegetação, bem como sua espacialização, foram feitas com o módulo "Earth trends modeler" (ETM) do programa Idrisi Taiga (Clark Labs, Worcester, MA, EUA). Para as análises estatísticas resultantes das tendências, utilizou-se mapa dos remanescentes de vegetação das Savanas Uruguaias juntamente com mapa de sistemas ecológicos da ecorregião das

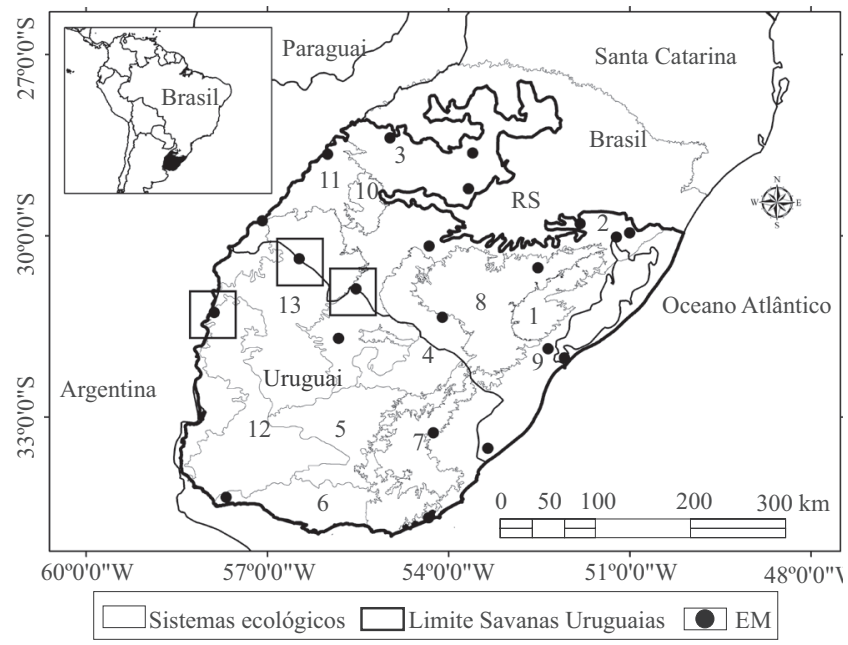

Figura 1. Sistemas ecológicos das diferentes unidades fitofisionômicas presentes na área de estudo e estações meteorológicas (EM) utilizadas. Adaptado de Hasenack et al. (2010). 1, Floresta estacional; 2, Campo misto de andropogoneas e compostas; 3, Campo com barba-de-bode; 4, Campo graminoso; 5, Campo com flechilhas do Cristalino Central; 6, Campo com flechilhas do Cristalino Meridional; 7, Campo misto do Cristalino Oriental; 8, Campo arbustivo; 9, Campo litorâneo; 10, Campo com areais; 11, Campo com espinilho; 12, Campo com flechilhas do Cristalino Ocidental; 13, Campo de solos rasos.
Savanas Uruguaias (Hasenack et al., 2010) (Figura 1). Todos os parâmetros foram extraídos sobre a máscara resultante do cruzamento entre a classe campo do mapa de remanescentes, os sistemas ecológicos e a tendência monotônica de Mann-Kendall, a 5\% de probabilidade, $\mathrm{p}<0,05$ para cada índice de vegetação. Dessa forma, apenas áreas campestres, em cada sistema ecológico, com tendência monotônica de Mann-Kendall, a 5\% de probabilidade, $\mathrm{p}<0,05$ foram analisadas.

Foram aplicadas técnicas de tendência linear (Jong \& Bruin, 2012) e monotônica de Mann-Kendall (Mann, 1945; Kendall, 1975), tendo-se tratado cada pixel no espaço como uma série temporal unidimensional de ciclos de 16 dias. Por meio de regressões e correlações da série com tendência linear, foram estimados o coeficiente de determinação $\left(\mathrm{R}^{2}\right)$ e o coeficiente de correlação do momento-produto de Pearson (r), pixel a pixel; no entanto, não foram aplicados testes para detectar se os resíduos da regressão foram normais. Assim, quanto à parametrização, foram usados modelos paramétricos (regressão linear) e não paramétricos (Mann-Kendall), na investigação de tendências negativas dos índices de vegetação.

No modelo de tendência monotônica de MannKendall, foram estimados os coeficientes de correlação de Mann-Kendall (S) e o tau ( $\tau$ ), dados pelas equações (Mann, 1945; Kendall, 1975):

$$
\begin{gathered}
S=\sum_{i=1}^{n-1} \sum_{j=i+1}^{n} \operatorname{sinal}\left(x_{i}-x_{j}\right), \\
\text { sinal }\left(x_{i}-x_{j}\right)=\left\{\begin{array}{l}
1, \text { se }\left(x_{i}-x_{j}\right)<0 \\
0, \text { se }\left(x_{i}-x_{j}\right)=0, \\
-1, \text { se }\left(x_{i}-x_{j}\right)>0
\end{array}\right.
\end{gathered}
$$

em que $\mathrm{S}$ é o coeficiente de correlação de MannKendall; $\mathrm{x}_{\mathrm{i}}$ e $\mathrm{x}_{\mathrm{j}}$ são os dados estimados da sequência de valores; e n é o número de elementos da série temporal.

Também foi utilizada a equação: $\tau=2 \mathrm{~s} /[\mathrm{n}(\mathrm{n}-1)]$, em que $\tau$ é o tau de Mann-Kendall e s é o sinal.

Para calcular a significância de Mann-Kendall (Mann, 1945; Kendall, 1975), obteve-se a estatística S do teste parametrizado $(\mathrm{Z})$, dado por:

$$
\mathrm{Z}=\left\{\begin{array}{cc}
\frac{\mathrm{s}-1}{\sqrt{\operatorname{Var}(\mathrm{S})}}, & \text { para } \mathrm{s}>0 \\
0, & \text { para } \mathrm{s}=0 \\
\frac{\mathrm{s}+1}{\sqrt{\operatorname{Var}(\mathrm{S})}}, & \text { para } \mathrm{s}<0
\end{array}\right.
$$


em que $\operatorname{Var}(\mathrm{S})$ é a variância e $\mathrm{S}$ é o coeficiente de correlação de Mann-Kendall.

Um valor positivo de $\mathrm{Z}$ indica tendência crescente, e um valor negativo, tendência decrescente. Para testar a tendência monotônica crescente ou decrescente no nível de significância de $\mathrm{p}$, a hipótese nula será rejeitada se o valor absoluto de $Z$ for maior que $\left(Z_{1}-p / 2\right)$, com uso da tabela da distribuição normal cumulativa padrão (Neeti et al., 2011) e da equação: $p=2[1-\phi(|Z|)]$, em que $\phi(|Z|)$ é a função de distribuição cumulativa de uma variação do desvio-padrão.

A tendência monotônica de Mann-Kendall, a 5\% de probabilidade, foi utilizada para análise e extração dos valores paramétricos das tendências em 81,9 e $74,8 \%$ das áreas de cobertura de campos, com uso da série de NDVI e EVI, respectivamente.

O valor médio da umidade do solo foi calculado a partir da série diária dos dados do CFSR, após o processamento, pixel a pixel, para as séries temporais de profundidades de 40 e $100 \mathrm{~cm}$. Além disso, ao se considerar apenas áreas de campo até $10 \mathrm{~km}$ de distância de cada estação meteorológica, foram extraídos os valores médios de NDVI e EVI. Os valores de precipitação pluvial diária foram agrupados em períodos de 16 dias totais acumulados, para estarem temporalmente compatíveis com as séries de índices de vegetação.

\section{Resultados e Discussão}

As estatísticas extraídas de áreas de campo, com paleta de tonalidades de cores realçada em função dos valores médios das variáveis (Tabelas 1 e 2), corresponderam a intervalos de $-0,25<\tau<0$, para o tau de Mann-Kendall, e de $-0,3<\mathrm{r}<0$ para a correlação (Figura 2). Assim, em gráficos de perfis, as duas variáveis foram apresentadas na mesma escala de frequência temporal, o que permitiu observar a evolução dos perfis de forma integrada.

Os interceptos, em unidades de índices de vegetação médios, apresentaram maiores valores para o NDVI do que para o EVI, na ordem de $\alpha_{\mathrm{NDVI}}=0,6$ e $\alpha_{\mathrm{EVI}}=0,4$, com variabilidade a partir da segunda casa decimal entre cada sistema ecológico, nos respectivos índices (Tabela 1). Em ambos os índices, os maiores valores médios coincidiram com os padrões de campo de vegetação mais arbustiva, e os menores valores, com as formações vegetacionais de porte baixo ou com os solos rasos ou de baixa fertilidade, de modo que os índices calculados de forma diferente diferiram em saturação. Esta é uma característica esperada, já que o NDVI apresenta saturação nos valores altos, o que o torna pouco sensível à detecção de variações no aumento da biomassa vegetal a partir de uma determinada fase de crescimento das plantas. O EVI, que se caracteriza por ser um NDVI aperfeiçoado, ao

Tabela 1. Parâmetros do modelo linear $(\alpha+\beta \mathrm{t})$, em que $\alpha$ é o intercepto, $\beta$ é a declividade e té o tempo em múltiplo de 16 dias para a série de 2000 a 2011, estimados da tendência das séries dos índices de vegetação NDVI e EVI, extraídos em áreas de campos, em 13 sistemas ecológicos.

\begin{tabular}{|c|c|c|c|c|}
\hline \multirow{2}{*}{ Sistema } & \multicolumn{2}{|l|}{ NDVI } & \multicolumn{2}{|l|}{ EVI } \\
\hline & $(\alpha+\beta \mathrm{t})_{\mathrm{NDVI}}$ & $\mathrm{R}^{2}$ & $(\alpha+\beta \mathrm{t})_{\mathrm{EVI}}$ & $\mathrm{R}^{2}$ \\
\hline Campo arbustivo (médio) & $0,691-0,00020 t$ & 0,042 & $0,448-0,00020 \mathrm{t}$ & 0,039 \\
\hline Campo com areais & $0,627-0,00013 t$ & 0,017 & $0,390-0,00017 \mathrm{t}$ & 0,026 \\
\hline Campo com barba-de-bode & $0,678-0,00014 t$ & 0,004 & $0,432-0,00018 \mathrm{t}$ & 0,007 \\
\hline Campo com espinilho (médio) & $0,670-0,00025 \mathrm{t}$ & 0,015 & $0,411-0,00022 \mathrm{t}$ & 0,015 \\
\hline Campo com flechilhas do Cristalino Central & $0,669-0,00024 t$ & 0,038 & $0,438-0,00022 \mathrm{t}$ & 0,035 \\
\hline Campo com flechilhas do Cristalino Meridional & $0,665-0,00021 \mathrm{t}$ & 0,026 & $0,416-0,00017 \mathrm{t}$ & 0,018 \\
\hline Campo com flechilhas do Cristalino Ocidental & $0,662-0,00026 \mathrm{t}$ & 0,024 & $0,419-0,00023 \mathrm{t}$ & 0,024 \\
\hline Campo de solos rasos & $0,655-0,00027 \mathrm{t}$ & 0,054 & $0,416-0,00026 \mathrm{t}$ & 0,079 \\
\hline Campo graminoso & $0,683-0,00023 t$ & 0,030 & $0,441-0,00021 \mathrm{t}$ & 0,024 \\
\hline Campo litorâneo & $0,664-0,00016 \mathrm{t}$ & 0,005 & $0,402-0,00009 t$ & 0,003 \\
\hline Campo misto de andropogôneas e compostas & $0,676-0,00018 \mathrm{t}$ & 0,021 & $0,429-0,00019 t$ & 0,021 \\
\hline Campo misto do Cristalino Oriental & $0,686-0,00020 t$ & 0,032 & $0,453-0,00020 \mathrm{t}$ & 0,028 \\
\hline Floresta estacional & $0,699-0,00005 t$ & 0,007 & $0,437-0,00011 t$ & 0,006 \\
\hline
\end{tabular}


incluir a banda do azul, não satura tão facilmente como o NDVI em áreas com grande quantidade de clorofila, mas corrige distorções na luz refletida causada por partículas no ar e na componente de resposta do solo abaixo da vegetação (Huete et al., 2002).

Em todos os índices, em todos os sistemas ecológicos, a declividade da tendência linear foi negativa, isto é, houve tendência de diminuição dos índices de vegetação, na mesma ordem de grandeza, que decresceram ligeiramente da vegetação menos arbustiva para a mais arbustiva. As taxas de declividade, no entanto, foram muito baixas e situaram-se em torno de $\beta=0,00002$, um valor baixo, mas significativo (Tabela 1). Os coeficientes de determinação quase nulos, encontrados em grande parte dos campos, relacionam-se a baixos valores de declividade da tendência linear e podem mostrar as características de sazonalidade destes padrões de cobertura, os quais apresentam grande variabilidade intra-anual associada às estações do ano bem definidas nessa região (Kuplich et al., 2013). Portanto, as séries de índices de vegetação apresentaram comportamento quase estacionário, mas com evidências de diminuição, cujas tendências não podem ser detectadas essencialmente por meio de ajuste linear em todas as áreas, como observado por Leeuwen et al. (2013) em diferentes escalas.

Tabela 2. Estimativas médias do tau de Mann-Kendall $(\tau)$ e desvios-padrão $(\sigma)$ da tendência monotônica de Mann-Kendall para NDVI e EVI, nos diferentes sistemas ecológicos.

\begin{tabular}{lccccc}
\hline Sistema $^{(1)}$ & \multicolumn{2}{c}{ NDVI } & & \multicolumn{2}{c}{ EVI } \\
\cline { 2 - 3 } \cline { 6 - 7 } & $\tau$ & $\sigma$ & & $\tau$ & $\sigma$ \\
\hline Campo arbustivo (médio) & $-0,153$ & 0,072 & & $-0,152$ & 0,059 \\
Campo com areais & $-0,106$ & 0,097 & & $-0,141$ & 0,077 \\
Campo com barba-de-bode & $-0,118$ & 0,045 & & $-0,142$ & 0,051 \\
Campo com espinilho (médio) & $-0,156$ & 0,058 & & $-0,160$ & 0,063 \\
Campo com flechilhas do CC & $-0,153$ & 0,054 & & $-0,152$ & 0,055 \\
Campo com flechilhas do CM & $-0,143$ & 0,065 & & $-0,122$ & 0,086 \\
Campo com flechilhas do CO & $-0,151$ & 0,072 & & $-0,161$ & 0,068 \\
Campo de solos rasos & $-0,173$ & 0,045 & & $-0,207$ & 0,057 \\
Campo graminoso & $-0,160$ & 0,058 & & $-0,146$ & 0,068 \\
Campo litorâneo & $-0,126$ & 0,090 & & $-0,080$ & 0,115 \\
Campo misto de AC & $-0,130$ & 0,085 & & $-0,142$ & 0,065 \\
Campo misto do Cristalino Oriental & $-0,148$ & 0,058 & & $-0,146$ & 0,058 \\
Floresta estacional & $-0,057$ & 0,129 & & $-0,108$ & 0,075 \\
\hline
\end{tabular}

${ }^{(1)} \mathrm{CC}$, Cristalino Central; CM, Cristalino Meridional; CO, Cristalino Ocidental; AC, andropogôneas e compostas.
No geral, o coeficiente de correlação do momentoproduto de Pearson (r) e o índice de tau de MannKendall $(\tau)$ apresentaram padrões espaciais semelhantes para NDVI e EVI, com valores médios de $r=-0,22$ e $\tau=0,12$, tendo atingido valores negativos maiores com a série de EVI (Figura 2). Além disso, especialmente na tendência monotônica de Mann-Kendall da série de EVI, também verificou-se aumento da tendência negativa no sentido leste a oeste na região, com destaque para os valores obtidos no campo de solos rasos, no seu contato com a porção norte do campo com espinilho e ao longo de toda a borda oeste do campo arbustivo. A tendência monotônica evidenciou valores negativos no $\tau$, com indicativo de tendência não linear decrescente, consistente em grande parte dos campos e em ambos os índices de vegetação (Tabela 2), à exceção do $\tau$ para o EVI, nos campos do litoral do Oceano Atlântico, que apresentou valores crescentes ou nulos. Em todos os casos, houve pequena variabilidade interna, observada nos baixos valores de desviospadrão médios estimados. Os sinais negativos de $\mathrm{r}$ e $\tau$ corroboram os resultados de Jong et al. (2011), que calcularam tendências monotônicas negativas globais ao utilizar dados de índices de vegetação derivados do "advanced very high resolution radiometer" (AVHRR) do produto "global inventory modeling and mapping studies" (Gimms), na série temporal de 1981-2006.

Constatou-se nítido sinal de diminuição do vigor vegetativo, associado ao campo de solos rasos. Nesses locais, a pecuária extensiva tem avançado substancialmente sobre os campos nos últimos anos (Paruelo et al., 2006), e o sobrepastoreio pode estar impactando negativamente a área.

A área de maior sinal negativo também apresentou os menores valores médios para as séries de índices de umidade do solo, nas profundidades de 40 e $100 \mathrm{~cm}$ (Figura 3). Este resultado é indicativo de que o campo de solos rasos apresenta a menor capacidade de retenção de água, comparativamente aos outros sistemas ecológicos, com exceção de uma área menor ao sul, em contato com o campo misto do Cristalino Oriental e o campo arbustivo. Provavelmente, esta condição, somada à intensificação da demanda evapotranspirativa da vegetação nos meses de verão na região (Castaño et al., 2011), potencializa o deficit hídrico nos campos de solos rasos, o que pode intensificar e prolongar os efeitos de estiagens pela diminuição da capacidade de água disponível 

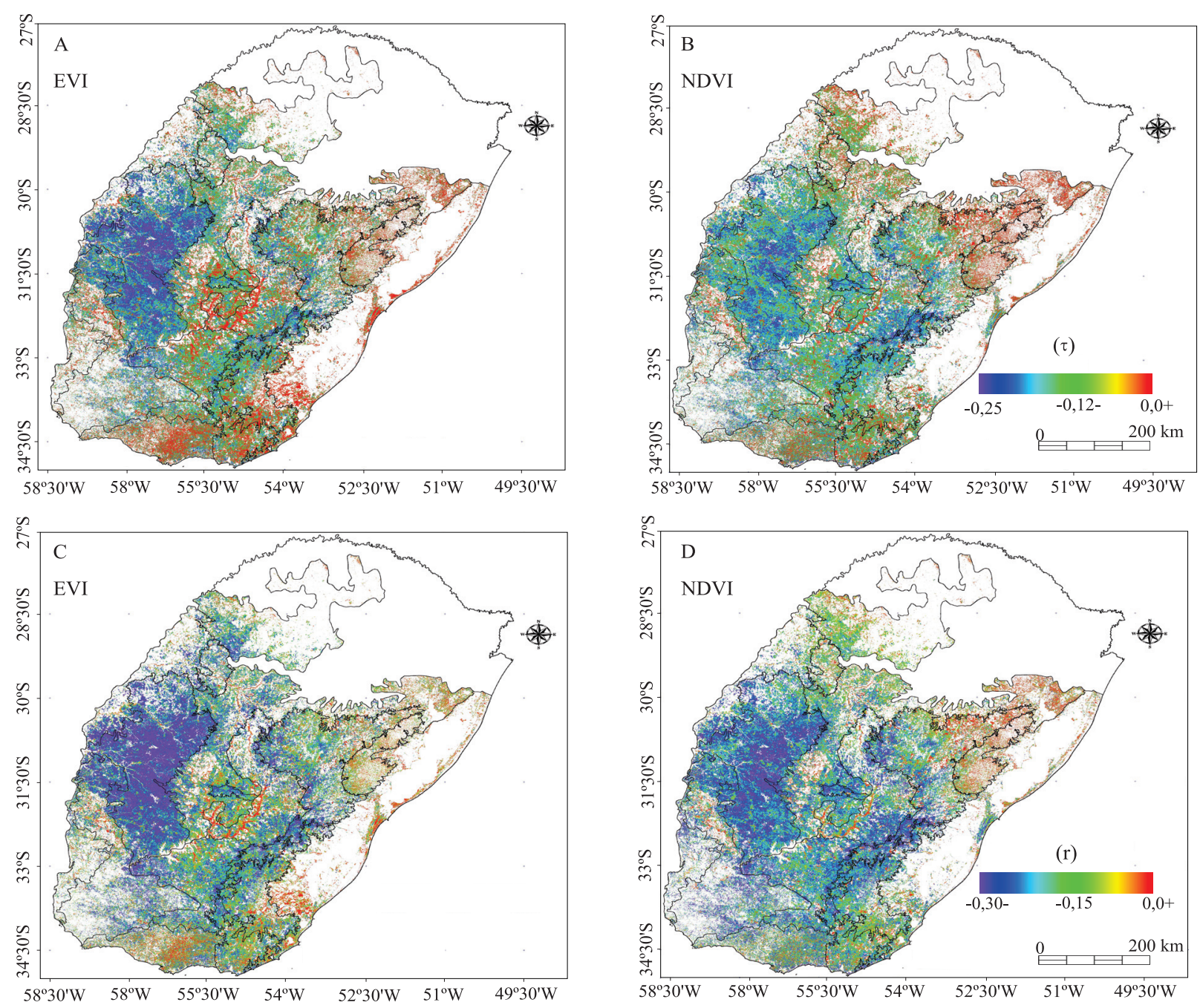

Figura 2. Distribuição espacial do tau de Mann-Kendall $(\tau)$ da tendência monotônica (A e B) e do coeficiente de correlação (r) da tendência linear (C e D), para NDVI e EVI, a 5\% de probabilidade. Resultados de parâmetros extraídos em cada sistema ecológico: lineares de intercepto $(\alpha)$, declividade $(\beta)$ e coeficiente de determinação $\left(\mathrm{R}^{2}\right)$ (Tabela 1); e não lineares de tau de Mann-Kendall e desvio-padrão médios (Tabela 2).
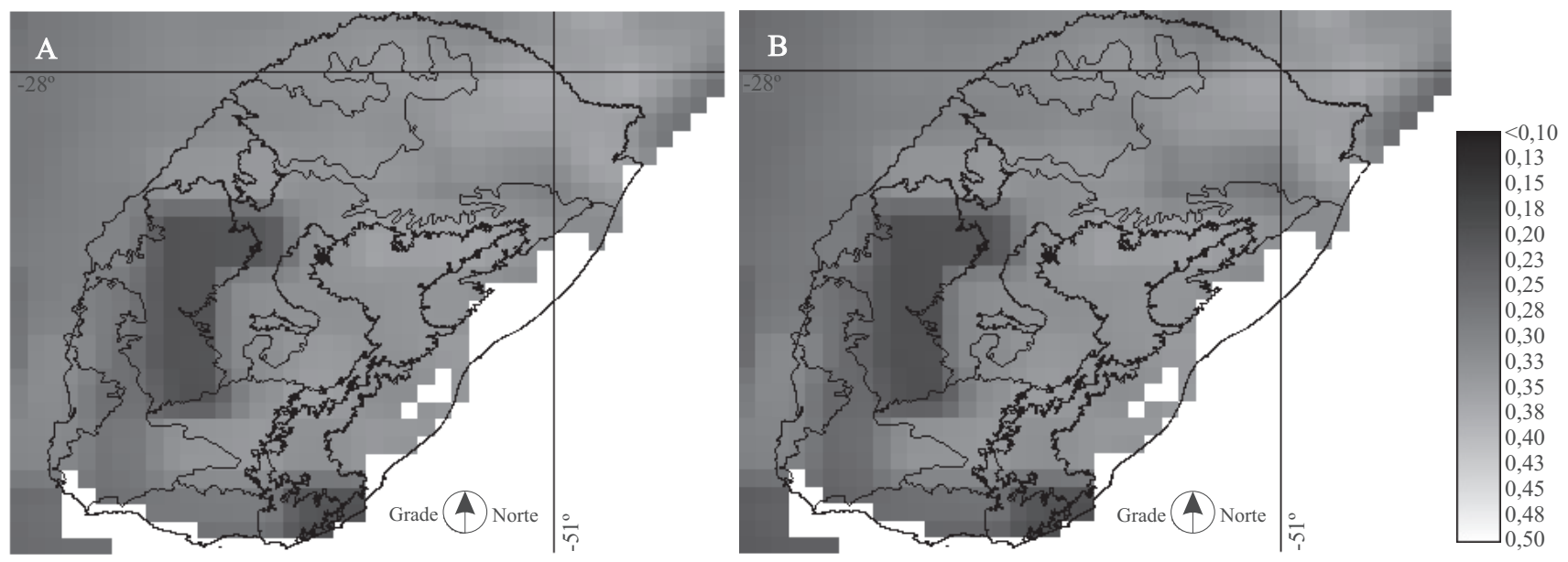

Figura 3. Índice de umidade do solo (adimensional, de 0 a 1), calculada para séries com profundidade de $40 \mathrm{~cm}$ (A) e $100 \mathrm{~cm}$ (B). 
(Zaitchik et al., 2013). Dessa forma, a resposta da interação entre solo-atmosfera, estabelecida pela resposta da umidade do solo, mostra a suscetibilidade ao estresse hídrico ao qual as pastagens da região estão sujeitas.

Os valores de precipitação pluvial e índices de vegetação diminuíram ao longo do tempo, em todas as variáveis e estações meteorológicas. Os coeficientes de declividade das retas, ajustadas sobre as séries de índicesdevegetaçãoeprecipitaçãopluvial, apresentaram valores negativos e padrões similares, cujas retas decresceram de forma quase paralela (Figura 4). Essas variáveis evoluíram com taxa linear similar, o que indica possíveis relações entre elas, observadas nas tendências decrescentes.

Contudo, na série analisada (2000-2011), os decréscimos encontrados podem estar restritos a efeitos ocorridos no período e não podem ser caracterizados em um contexto de tendências climáticas, uma vez que estudos de longo prazo, atualmente, apontam aumento da precipitação pluvial no Sul do Brasil (Boschi et al., 2011) e no Uruguai (Deschamps et al., 2003). Esses resultados podem parecer contraditórios com os do presente trabalho; porém, se referem a períodos diferentes, e, portanto, o padrão pode ser distinto. De 2000 em diante, houve diminuição da precipitação pluvial na região e os índices de vegetação apresentaram a mesma tendência. Além disso, o efeito da diminuição na produção de biomassa pode ter sido potencializado em anos extremamente secos, em razão do manejo, pois muitas das pastagens da região estão sujeitas ao sobrepastoreio (Carvalho et al., 2006; Berreta, 2009), o que causa efeito cumulativo para as temporadas seguintes.

$\mathrm{Na}$ série analisada, a variabilidade interanual da precipitação pode ter sido induzida pelo fenômeno Enos, especialmente pela ocorrência de vários eventos moderados de La Niña, cujo efeito observado na região foi de estiagens repetidas (Rosemback et al., 2010). Estudos já apontaram a resposta da vegetação aos efeitos cumulativos da ocorrência destes fenômenos nos mesmos campos (Jong et al., 2011).

Enquanto análises de longo prazo das tendências climáticas buscam avaliar as mudanças no clima, o presente trabalho evidencia a necessidade de se executar análises de curto prazo, dada a dinâmica da resposta da vegetação campestre à variabilidade climática.
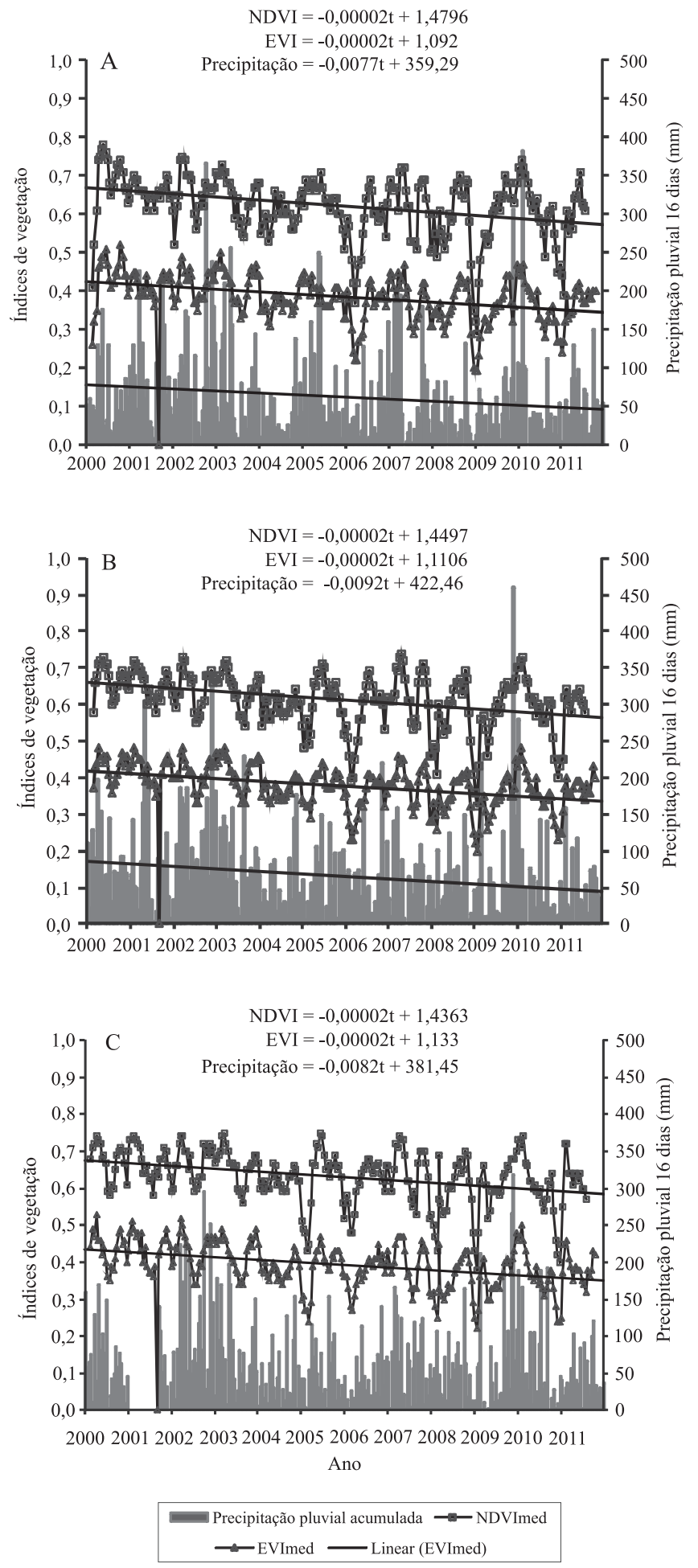

Figura 4. Perfis temporais médios de NDVI e EVI sobre pastagens, significativos a $5 \%$ de probabilidade, no entorno das estações meteorológicas de: A, Salto, Uruguai; B, Quarai, Rio Grande do Sul; e C, Santana do Livramento, Rio Grande do Sul; e padrão temporal de precipitação pluvial acumulada, para períodos de 16 dias, de 2000 a 2011. 


\section{Conclusões}

1. Os índices EVI e NDVI do sensor Modis possibilitam detectar variações temporais na vegetação de diferentes formações campestres do Bioma Pampa no Rio Grande do Sul e no Uruguai.

2. As tendências negativas significativas observadas nas séries temporais de imagens NDVI e EVI indicam alterações no padrão de cobertura vegetal das formações campestres no Bioma Pampa, com redução no vigor vegetativo.

\section{Agradecimentos}

À Coordenação de Aperfeiçoamento de Pessoal de Nível Superior (Capes), pela bolsa de estudo; e ao Eduardo Gelcer, pela organização dos dados de precipitação pluvial.

\section{Referências}

BERRETA, E. Algunos aspectos sobre la biodiversidad de los campos naturales. Revista INIA Uruguay, v.20, p.21-25, 2009.

BOSCHI, R.S.; OLIVEIRA, S.R. de M.; ASSAD, E.D. Técnicas de mineração de dados para análise da precipitação pluvial decenal no Rio Grande do Sul. Engenharia Agrícola, v.31, p.1189-1201, 2011. DOI: 10.1590/S0100-69162011000600016.

CARVALHO, P.C. de F.; FISHER, V.; SANTOS, D.T. dos; RIBEIRO, A.M.L.; QUADROS, F.L.F. de; CASTILHOS, Z.M.S.; POLI, C.H.E.C.; MONTEIRO, A.L.G.; NABINGER, C.; GENRO, T.C.M.; JACQUES, A.V.A. Produção animal no Bioma Campos Sulinos. Brazilian Journal of Animal Science, v.35, p.156-202, 2006.

CASTAÑO, J.P.; GIMENEZ, A.; CERONI, M.; FUREST, J.; AUNCHAYNA, R. Caracterización agroclimática del Uruguay 1980-2009. Montevideo: INIA, 2011. 40p. (INIA. Serie técnica, 193).

CRAWSHAW, D.; DALL'AGNOL, M.; CORDEIRO, J.L.P.; HASENACK, H. Caracterização dos campos sul-rio-grandenses: uma perspectiva da ecologia da paisagem. Boletim Gaúcho de Geografia, n.33, p.233-252, 2007.

DESCHAMPS, J.R.; OTERO, O.; TONNI, E.P. Cambio climático en la pampa bonaerense: las precipitaciones desde los siglos XVIII al XX. Buenos Aires: Universidad de Belgrano, 2003. (Universidad de Belgrano. Documentos de trabajo, 109).

EK, M.B.; MITCHELL, K.E.;LIN, Y.; ROGERS, E.; GRUNMANN, P.; KOREN, V.; GAYNO, G.; TARPLEY, J.D. Implementation of Noah land surface model advances in the National Centers for Environmental Prediction operational mesoscale Eta model. Journal of Geophysical Research: Atmospheres, v.108, p.12-15, 2003. DOI: 10.1029/2002JD003296.

FORKEL, M.; CARVALHAIS, N.; VERBESSELT, J.; MAHECHA, M.D.; NEIGH, C.S.R.; REICHSTEIN, M. Trend change detection in NDVI time series: effects of inter-annual variability and methodology. Remote Sensing, v.5, p.2113-2144, 2013. DOI: $10.3390 /$ rs5052113.

HASENACK, H.; WEBER, E.; BOLDRINI, I.I.; TREVISAN, R. Mapa de sistemas ecológicos da ecorregião das Savanas Uruguaias em escala 1:500.000 ou superior e relatório técnico descrevendo insumos utilizados e metodologia de elaboração do mapa de sistemas ecológicos. Porto Alegre: UFRGS, 2010.

HUETE, A.; DIDAN, K.; MIURA, T.; RODRIGUEZ, E.P.; GAO, X.; FERREIRA, L.G. Overview of the radiometric and biophysical performance of the MODIS vegetation indices. Remote Sensing of Environment, v.83, p.195-213, 2002. DOI: 10.1016/S00344257(02)00096-2.

HUETE, A; LIU, H.Q.; BATCHILY, K.; LEWEEN, W. A comparison of vegetation indices over a global set of TM images for EOS-MODIS. Remote Sensing of Environment, v.59, p.440451, 1997. DOI: 10.1016/S0034-4257(96)00112-5.

JACÓBSEN, L.O.; FONTANA, D.C.; SHIMABUKURO, Y.E. Efeitos associados a El Niño e La Niña na vegetação do Estado do Rio Grande do Sul observados através do NDVI/NOAA. Revista Brasileira de Meteorologia, v.19, p.129-140, 2004.

JAURENA, M.; FORMOSO, D.; MILLER, R.G.; REBUFFO, M. Campo natural: patrimonio del país y fundamento de la estabilidad productiva de la ganadería. Revista INIA Uruguay, v.32, p.30-35, 2013.

JI, L.; ZHANG, L.; WYLIE, B.K.; ROVER, J. On the terminology of the spectral vegetation index (NIR - SWIR)/(NIR+SWIR). International Journal of Remote Sensing, v.32, p.6901-6909, 2011. DOI: 10.1080/01431161.2010.510811.

JONG, R. de; BRUIN, S. de. Linear trends in seasonal vegetation time series and the modifiable temporal unit problem. Biogeosciences, v.9, p.71-77, 2012. DOI: 10.5194/bg-9-71-2012.

JONG, R. de; BRUIN, S. de; WIT, A. de; SCHAEPMAN, M.E.; DENT, D.L. Analysis of monotonic greening and browning trends from global NDVI time-series. Remote Sensing of Environment, v.115, p.692-702, 2011. DOI: 10.1016/j.rse.2010.10.011.

KENDALL, M.G. Rank correlation measures. London: Charles Griffin, 1975. 220p.

KUPLICH, T.M.; MOREIRA, A.; FONTANA, D.C. Série temporal de índice de vegetação sobre diferentes tipologias vegetais no Rio Grande do Sul. Revista Brasileira de Engenharia Agrícola e Ambiental, v.17, p.1116-1123, 2013. DOI: 10.1590/S141543662013001000014.

LEEUWEN, W.J.D. van; HARTFIELD, K.; MIRANDA, M.; MEZA, F.J. Trends and ENSO/AAO driven variability in NDVI derived productivity and phenology alongside the Andes Mountains. Remote Sensing, v.5, p.1177-1203, 2013. DOI: 10.3390/rs5031177.

LEEUWEN, W.J.D. van; ORR, B.J.; MARSH, S.E.; HERRMANN, S.M. Multi-sensor NDVI data continuity: uncertainties and implications for vegetation monitoring applications. Remote Sensing of Environment, v.100, p.67-81, 2006. DOI: 10.1016/j. rse.2005.10.002.

Pesq. agropec. bras., Brasília, v.48, n.9, p.1192-1200, set. 2013 DOI: $10.1590 / \mathrm{S} 0100-204 \mathrm{X} 2013000900002$ 
MANN, H.B. Nonparametric test against trend. Econometrica, v.13, p.245-259, 1945. DOI: 10.2307/1907187.

NEETI, N.; EASTMAN, J.R. A contextual Mann-Kendall approach for the assessment of trend significance in image time series. Transactions in GIS, v.15, p.599-611, 2011. DOI: 10.1111/j.14679671.2011.01280.x.

PARUELO, J.M.; GUERSCHMAN, J.P.; PIÑEIRO, G.; JOBBÁGY, E.G.; VERÓN, S.R.; BALDI, G.; BAEZA, S. Cambios en el uso de la tierra en Argentina y Uruguay: marcos conceptuales para su análisis. Agrociencia, v.10, p.47-61, 2006.

PILLAR, V. de P.; VÉLEZ, E. Extinção dos Campos Sulinos em unidades de conservação: um fenômeno natural ou um problema ético? Natureza e Conservação, v.8, p.84-86, 2010. DOI: 10.4322/ natcon.00801014.

RISSO, J.; RIZZI, R.; RUDORFF, B.F.T.; ADAMI, M.; SHIMABUKURO, Y.E.; FORMAGGIO, A.R.; EPIPHANIO, R.D.V. Índices de vegetação MODIS aplicados na discriminação de áreas de soja. Pesquisa Agropecuária Brasileira, v.47, p.13171326, 2012. DOI: 10.1590/S0100-204X2012000900017.

ROSEMBACK, R.; FERREIRA, N.J.; SHIMABUKURO, Y.E.; CONFORTE, J.C. Análise da dinâmica da cobertura vegetal na região Sul do Brasil a partir de dados MODIS/Terra. Revista Brasileira de Cartografia, v.62, p.401-416, 2010.
ROUSE, J.W.; HASS, R.H.; DEERING, D.W.; SCHELL, J.A. Monitoring the vernal advancement and retrogradation (green wave effect) of natural vegetation. College Station: Texas A\&M University, 1974. 87p. (Progress Report RSC 19782). Available at: $<$ http://ntrs.nasa.gov/archive/nasa/casi.ntrs.nasa. gov/19740004927_1974004927.pdf >. Accessed on: 23 Aug. 2012.

SAHA, S.; MOORTHI, S.; PAN, H.-L.; WU, X.; WANG, JIANDE; NADIGA, S.; TRIPP, P.; KISTLER, R.; WOOLLEN, J.; BEHRINGER, D.; LIU, H.; STOKES, D.; GRUMBINE, R.; GAYNO, G.; WANG, JUN; HOU, Y.-T.; CHUANG, H.-Y.; JUANG, H.-M.H.; SELA, J.; IREDELL, M.; TREADON, R.; KLEIST, D.; VAN DELST, P.; KEYSER, D.; DERBER, J.; EK, M.; MENG, J.; WEI, H.; YANG, R.; LORD, S.; DOOL, H.V.D.; KUMAR, A.; WANG, W.; LONG, C.; CHELLIAH, M.; XUE, Y.; HUANG, B.; SCHEMM, J.-K.; EBISUZAKI, W.; LIN, R.; XIE, P.; CHEN, M.; ZHOU, S.; HIGGINS, W.; ZOU, C.-Z.; LIU, Q.; CHEN, Y.; HAN, Y.; CUCURULL, L.; REYNOLDS, R.W.; RUTLEDGE, G.; GOLDBERG, M. The NCEP climate forecast system reanalysis. Bulletin of the American Meteorological Society, v.91, p.10151057, 2010. DOI: 10.1175/2010BAMS3001.1.

ZAITCHIK, B.F.; SANTANELLO, J.A.; KUMAR, S.V.; PETERSLIDARD, C.D. Representation of soil moisture feedbacks during drought in NASA unified WRF (NU-WRF). Journal of Hydrometeorology, v.14, p.360-367, 2013. DOI: 10.1175/ JHM-D-12-069.1.

Recebido em 24 de abril de 2013 e aprovado em 30 de agosto de 2013 